\title{
AGLER INTERPOLATION FAMILIES OF KERNELS
}

\author{
Michael T. Jury ${ }^{1}$, Greg Knese ${ }^{2}$ And Scott McCullough ${ }^{3}$
}

Abstract. An abstract Pick interpolation theorem for a family of positive semi-definite kernels on a set $X$ is formulated. The result complements those in [Ag] and [AM02] and will subsequently be applied to Pick interpolation on distinguished varieties [JKM].

Mathematics subject classification (2000): 47A57 (Primary), 47A20, 47L30 (Secondary). Keywords and phrases: reproducing kernel, Nevanlinna-Pick interpolation.

\section{REFERENCES}

[Ab] M. B. Abrahamse, The Pick interpolation theorem for finitely connected domains, Michigan Math. J. 26 (1979), no. 2, 195-203.

[AD] M. B. ABRAHAMSE AND RONALD Douglas, A class of subnormal operators related to multiplyconnected domains, Advances in Math. 19 (1976), no. 1, 106-148.

[Ag] JIM AGLER, Interpolation, unpublished manuscript.

[AM99] Jim AgLER AND John MCCARThy, Nevanlinna-Pick interpolation on the bidisk, J. Reine Angew. Math. 506 (1999), 191-204.

[AM00] Jim Agler and John McCarthy, Complete Nevanlinna-Pick kernels, J. Funct. Anal. 175 (2000), no. 1, 111-124.

[AM02] Jim Agler And John MCCARThy, Pick interpolation and Hilbert function spaces, Graduate Studies in Mathematics, 44. American Mathematical Society, Providence, RI, 2002. xx+308 pp. ISBN: 0-8218-2898-3.

[AM03] JIM AgLER AND JoHn MCCARTHY, Norm preserving extensions of holomorphic functions from subvarieties of the bidisk, Ann. of Math. (2) 157 (2003), no. 1, 289-312.

[AM05] Jim Agler And John McCarthy, Distinguished varieties, Acta Math. 194 (2005), no. 2, 133 153.

[B] JosePh BALL, Interpolation problems and Toeplitz operators on multiply connected domains, Integral Equations Operator Theory 4 (1981), no. 2, 172-184.

[BB] Joseph Ball and Vladimir Bolotnikov, Nevanlinna-Pick interpolation for Schur-Agler class functions on domains with matrix polynomial defining function in $\mathbb{C}^{n}$, New York J. Math. 11 (2005), 247-290.

[BCV] Joseph Ball, Kevin Clancey, And Victor Vinnikov, Concrete interpolation of meromorphic matrix functions on Riemann surfaces, Interpolation theory, systems theory and related topics (Tel Aviv/Rehovot, 1999), 137-156, Oper. Theory Adv. Appl., 134, Birkhäuser, Basel, 2002.

[BTV] JosePh BALL, TAVAN TRENT, AND VICTOR VINNIKOV, Interpolation and commutant lifting for multipliers on reproducing kernel Hilbert spaces, Operator theory and analysis (Amsterdam, 1997), 89-138, Oper. Theory Adv. Appl., 122, Birkhäuser, Basel, 2001.

[BL] David P. Blecher and Christian Le Merdy, Operator Algebras and their Modules. London Mathematical Society Monographs. New Series, 30. Oxford Science Publications. The Clarendon Press, Oxford University Press, Oxford, 2004.

[CLW] Brian Cole, Keith Lewis, And John Wermer, Pick conditions on a uniform algebra and von Neumann inequalities, J. Funct. Anal. 107 (1992), no. 2, 235-254.

[DPRS] Kenneth R. Davidson, Vern I. Paulsen, Mrinal Raghupathi, and Dinesh Singh, A constrained Nevanlinna-Pick interpolation problem, Indiana Univ. Math. J., 58, 2 (2009), 709-732. 
[DMM] Michael Dritschel, Stefania Marcantognini, and Scott McCullough, Interpolation in semigroupoid algebras, J. Reine Angew. Math. 606 (2007), 1-40.

[FF] CIPRIAN FoIAS AND ARTHUR FraZho, The commutant lifting approach to interpolation problems. Operator Theory: Advances and Applications, 44. Birkhäuser Verlag, Basel, 1990. xxiv+632 pp. ISBN: 3-7643-2461-9

[JK] MICHAEL JURY AND DAVID KRIBS, Ideal structure in free semigroupoid algebras from directed graphs, J. Operator Theory 53 (2005), no. 2, 273-302.

[JKM] Michael Jury, Greg Knese, and Scott McCullough, Pick interpolation on distinguished varieties, in progress.

[M] Scott McCullough, Nevanlinna-Pick type interpolation in a dual algebra, J. Funct. Anal. 135 (1996), no. 1, 93-131.

[MP] Scott McCullough And Vern Paulsen, $C^{*}$-envelopes and interpolation theory, Indiana Univ. Math. J. 51 (2002), no. 2, 479-505.

[MS] PaUl S. Muhly ANd BARUCh Solel, Schur Class Operator Functions and Automorphisms of Hardy Algebras, Documenta Mathematica 13 (2008) 365-411.

[Pa] VERn PAULSEN, Operator Algebras of Idempotents, J. Funct. Anal. 181 (2001), no. 2, 209-226.

[P] JAMES PICKERING, Test Functions in Constrained Interpolation, arXiv:0811.2191.

[Q] Peter Quiggin, For which reproducing kernel Hilbert spaces is Pick's theorem true?, Integral Equations and Operator Theory 16 (1993), no. 2, 244-266.

[R1] Mrinal RaghUPATHI, Nevanlinna-Pick interpolation for $\mathbb{C}+B H^{\infty}$, Integral Equations Operator Theory, 63, 1 (2009), 103-125.

[R2] Mrinal Raghupathi, Abrahamse's interpolation theorem and Fuchsian groups, J. Math. Anal. Appl., 355, 1 (2009), 258-276.

[S] Donald SARAson, Generalized interpolation in $H^{\infty}$, Trans. Amer. Math. Soc. 1271967179 203.

[S65] Donald Sarason, The $H^{p}$ spaces of an annulus, Mem. Amer. Math. Soc. no. 56196578 pp. 